\title{
Global existence and exponential stability of coupled Lamé system with distributed delay and source term without memory term
}

Salah Boulaaras ${ }^{1,2^{*}}$ and Nadjat Doudi ${ }^{3}$

"Correspondence:

s.Boularas@qu.edu.sa

${ }^{1}$ Department of Mathematics,

College of Sciences and Arts, ArRas,

Qassim University, Buraidah,

Kingdom of Saudi Arabia

${ }^{2}$ Laboratory of Fundamental and

Applied Mathematics of Oran

(LMFAO), University of Oran 1,

Ahmed Benbella, Oran, Algeria

Full list of author information is

available at the end of the article

\begin{abstract}
In this paper, we prove the global existence and exponential energy decay results of a coupled Lamé system with distributed time delay, nonlinear source term, and without memory term by using the Faedo-Galerkin method. In addition, an appropriate Lyapunov functional, more general relaxation functions, and some properties of convex functions are considered.
\end{abstract}

MSC: 35L90; 74G25; 35B40; 26A51

Keywords: Viscoelastic term; Delay function; Lyapunov functional; Distributed delay; Exponential decay

\section{Introduction}

In this work, we study the following Lamé system in $\Omega \times \mathbb{R}^{+}$:

$$
\left\{\begin{array}{l}
u_{t t}-\Delta_{e} u+k_{1} u_{t}+\int_{\tau_{1}}^{\tau_{2}} \mu_{1}(\varrho) u_{t}(x, t-\varrho) d \varrho=f_{1}(u, v), \\
v_{t t}-\Delta_{e} v+k_{2} v_{t}+\int_{\tau_{1}}^{\tau_{2}} \mu_{2}(\varrho) v_{t}(x, t-\varrho) d \varrho=f_{2}(u, v), \\
u(x, t)=v(x, t)=0 \quad \text { on } \partial \Omega \times \mathbb{R}^{+}, \\
u(x, 0)=u_{0}(x), \quad v(x, 0)=v_{0}(x), \quad u_{t}(x, 0)=u_{1}(x), \\
v_{t}(x, 0)=v_{1}(x), \quad x \in \Omega, \\
\left(u_{t}(x,-t), v_{t}(x,-t)\right)=\left(f_{0}(x, t), g_{0}(x, t)\right) \quad \text { in } \Omega \times\left(0, \tau_{2}\right),
\end{array}\right.
$$

where $\Omega$ is a bounded domain in $\mathbb{R}^{n}(n=1,2,3)$ with smooth boundary $\partial \Omega$. The elasticity differential operator $\Delta_{e}$ is given by

$$
\Delta_{e} u=\mu \Delta u+(\mu+\lambda) \nabla(\operatorname{div} u),
$$

and the Lamé constants $\mu$ and $\lambda$ satisfy the following conditions:

$$
\mu>0, \quad \mu+\lambda>0 .
$$

C) The Author(s) 2020. This article is licensed under a Creative Commons Attribution 4.0 International License, which permits use, sharing, adaptation, distribution and reproduction in any medium or format, as long as you give appropriate credit to the original author(s) and the source, provide a link to the Creative Commons licence, and indicate if changes were made. The images or other third party material in this article are included in the article's Creative Commons licence, unless indicated otherwise in a credit line to the material. If material is not included in the article's Creative Commons licence and your intended use is not permitted by statutory regulation or exceeds the permitted use, you will need to obtain permission directly from the copyright holder. To view a copy of this licence, visit http://creativecommons.org/licenses/by/4.0/. 
The parameters $k_{1}, k_{2}, \tau_{1}$, and $\tau_{2}$ are positive constants, with $\tau_{1}<\tau_{2}$. The functions $\mu_{1}, \mu_{2}$ : $\left[\tau_{1}, \tau_{2}\right] \rightarrow \mathbb{R}$ are bounded. The functions $f_{1}(u, v)$ and $f_{2}(u, v)$, which represent the source terms, will be specified later.

After several authors have studied the problems of coupled systems and hyperbolic systems, their stability is associated with velocities and is proven under some given conditions (see, for example, [1-11]). In recent years, several authors have been interested in studying the existence and stability for Lamé systems, we refer to [12-14]. The Lamé system with localized nonlinear damping and a general decay result of energy have been considered by some recent works (see, for example, [12, 14], and [15]). Bchatnia et al. in [16] investigated Lamé systems with past history. Then, Taouaf et al. in [17] established the well-posedness and asymptotic stability for the Lamé system with internal distributed delay.

Beniani et al. [13] proved the well-posedness and exponential stability of the following coupled Lamé system:

$$
\left\{\begin{array}{l}
u_{t t}+\alpha v-\Delta_{e} u+\int_{0}^{t} g_{1}(t-s) \Delta u(s) d s-\mu_{1} \Delta u_{t}=0 \\
v_{t t}+\alpha u-\Delta_{e} v+\int_{0}^{t} g_{2}(t-s) \Delta v(s) d s-\mu_{2} \Delta v_{t}=0
\end{array}\right.
$$

After that, Baowei et al. in [18] considered the same problem with more general assumption on the relaxation functions. They established an explicit and general decay result, which are optimal, to the system.

Boulaaras et al. in [14] considered the previous problem with a source term, where under some suitable conditions on the initial data and the relaxation functions, they proved an asymptotic stability result of global solution taking into account that the kernel is not necessarily decreasing.

In the present work, we prove the existence and general decay results of problem (1.1) with respect to the presence of distributed term delay in order to ensure fast stability under some given conditions. We establish the exponential energy decay results to the system by using an appropriate Lyapunov functional.

This paper is organized as follows. In the second section, we give some preliminaries related to problem (1.1). In Sect. 3, we prove the global existence by using Faedo-Galerkin method. In the fourth section, we prove our main result of exponential energy decay.

\section{Preliminaries}

In this section, we provide some materials and necessary assumptions which we need in the proof of our results. We use the standard Lebesgue and Sobolev spaces with their scaler products and norms. For simplicity, we would write $\|\cdot\|$ instead of $\|\cdot\|_{2}$.

(A1) For the source terms $f_{1}$ and $f_{2}$, we take

$$
f_{1}(u, v)=\alpha|u+v|^{p-1}(u+v)+\beta|u|^{\frac{p-3}{2}} u|v|^{\frac{p+1}{2}}, \quad \forall(u, v) \in\left[\mathbb{R}^{n}\right]^{2}
$$

and

$$
f_{2}(u, v)=\alpha|u+v|^{p-1}(u+v)+\beta|v|^{\frac{p-3}{2}} v|u|^{\frac{p+1}{2}}, \quad \forall(u, v) \in\left[\mathbb{R}^{n}\right]^{2},
$$

with $\alpha, \beta>0$. Clearly,

$$
u f_{1}(u, v)+v f_{2}(u, v)=(p+1) F(u, v), \quad \forall(u, v) \in\left[\mathbb{R}^{n}\right]^{2},
$$


where

$$
F(u, v)=\frac{1}{(p+1)}\left[\alpha|u+v|^{p+1}+2 \beta|u v|^{\frac{p+1}{2}}\right], \quad \forall(u, v) \in\left[\mathbb{R}^{n}\right]^{2},
$$

and

$$
f_{1}(u, v)=\frac{\partial F}{\partial u}, \quad f_{2}(u, v)=\frac{\partial F}{\partial v} .
$$

(A2)

$$
\text { If } n=1,2 ; \quad p \geq 3, \quad \text { if } n=3 ; \quad p=3 .
$$

So, we have the embedding

$$
H_{0}^{1}(\Omega) \hookrightarrow L^{q}(\Omega) \quad \text { for } 2 \leq q \leq \frac{2 n}{n-2} \text { if } n \geq 3 \quad \text { or } \quad q \geq 2 \quad \text { if } n=1,2
$$

and

$$
L^{r} \hookrightarrow L^{q} \quad \text { for } q<r .
$$

Let $c_{s}$ be the same embedding constant, so we have

$$
\|v\|_{q} \leq c_{s}\|\nabla v\|_{2}, \quad\|v\|_{q} \leq c_{s}\|v\|_{r} \quad \text { for } v \in H_{0}^{1}(\Omega) .
$$

As in many papers, we introduce the following new variables:

$$
\left\{\begin{array}{l}
z(x, \rho, \varrho, t)=u_{t}(x, t-\varrho \rho) \\
y(x, \rho, \varrho, t)=v_{t}(x, t-\varrho \rho)
\end{array}\right.
$$

then we obtain

$$
\left\{\begin{array}{l}
\varrho z_{t}(x, \rho, \varrho, t)+z_{\rho}(x, \rho, \varrho, t)=0 \\
z(x, 0, \varrho, t)=u_{t}(x, t)
\end{array}\right.
$$

and

$$
\left\{\begin{array}{l}
\varrho y_{t}(x, \rho, \varrho, t)+y_{\rho}(x, \rho, \varrho, t)=0, \\
y(x, 0, \varrho, t)=v_{t}(x, t) .
\end{array}\right.
$$

Consequently, problem (1.1) is equivalent to

$$
\left\{\begin{array}{l}
u_{t t}-\Delta_{e} u+k_{1} u_{t}+\int_{\tau_{1}}^{\tau_{2}} \mu_{1}(\varrho) z(x, 1, \varrho, t) d \varrho=f_{1}(u, v), \\
v_{t t}-\Delta_{e} v+k_{2} v_{t}+\int_{\tau_{1}}^{\tau_{2}} \mu_{2}(\varrho) y(x, 1, \varrho, t) d \varrho=f_{2}(u, v), \\
\varrho z_{t}(x, \rho, \varrho, t)+z_{\rho}(x, \rho, \varrho, t)=0 \\
\varrho y_{t}(x, \rho, \varrho, t)+y_{\rho}(x, \rho, \varrho, t)=0
\end{array}\right.
$$


with the initial data and boundary conditions

$$
\left\{\begin{array}{l}
(u(x, 0), v(x, 0))=\left(u_{0}(x), v_{0}(x)\right) \quad \text { in } \Omega, \\
\left(u_{t}(x, 0), v_{t}(x, 0)\right)=\left(u_{1}(x), v_{1}(x)\right) \quad \text { in } \Omega, \\
\left(u_{t}(x,-t), v_{t}(x,-t)\right)=\left(f_{0}(x, t), g_{0}(x, t)\right) \quad \text { in } \Omega \times\left(0, \tau_{2}\right), \\
u(x, t)=v(x, t)=0 \quad \text { in } \partial \Omega \times(0, \infty), \\
(z(x, \rho, \varrho, 0), y(x, \rho, \varrho, 0))=\left(f_{0}(x, \rho \varrho), g_{0}(x, \rho \varrho)\right) \quad \text { in } \Omega \times(0,1) \times\left(0, \tau_{2}\right),
\end{array}\right.
$$

where

$$
(x, \rho, \varrho, t) \in \Omega \times(0,1) \times\left(\tau_{1}, \tau_{2}\right) \times(0, \infty) .
$$

The energy associated with problem (2.8) is defined by

$$
\begin{aligned}
E(t)= & \frac{1}{2}\left[\left\|u_{t}\right\|^{2}+\left\|v_{t}\right\|^{2}+\mu\left(\|\nabla u\|^{2}+\|\nabla v\|^{2}\right)+(\lambda+\mu)\left(\|\operatorname{div} u\|^{2}+\|\operatorname{div} v\|^{2}\right)\right] \\
& +\frac{1}{2} \int_{\Omega} \int_{0}^{1} \int_{\tau_{1}}^{\tau_{2}} \varrho\left[\left|\mu_{1}(\varrho)\left\|\left.z(x, \rho, \varrho, t)\right|^{2}+\left|\mu_{2}(\varrho) \| y(x, \rho, \varrho, t)\right|^{2}\right] d \varrho d \rho d x\right.\right. \\
& -\int_{\Omega} F(u, v) d x .
\end{aligned}
$$

First, we prove in the following theorem, the result of energy identity.

\section{Lemma 2.1 Assume that}

$$
\int_{\tau_{1}}^{\tau_{2}}\left|\mu_{i}(\varrho)\right| d \varrho<k_{i}, \quad i=1,2 .
$$

Then the energy modified defined by (2.10) satisfies, along the solution $(u, v, z, y)$ of $(2.8)$, the estimate

$$
\begin{aligned}
\frac{d}{d t} E(t) & \leq-\left[k_{1}-\left(\int_{\tau_{1}}^{\tau_{2}}\left|\mu_{1}(\varrho)\right| d \varrho\right)\right]\left\|u_{t}\right\|^{2}-\left[k_{2}-\left(\int_{\tau_{1}}^{\tau_{2}}\left|\mu_{2}(\varrho)\right| d \varrho\right)\right]\left\|v_{t}\right\|^{2} \\
& \leq 0 .
\end{aligned}
$$

Proof First multiplying equation (2.8) $)_{1}$ by $u_{t}$ and integrating by parts over $\Omega$, we obtain

$$
\begin{aligned}
\frac{1}{2} \frac{d}{d t} & {\left[\left\|u_{t}\right\|^{2}+\mu\|\nabla u\|^{2}+(\lambda+\mu)\|\operatorname{div} u\|^{2}\right]+k_{1}\left\|u_{t}\right\|^{2} } \\
& +\int_{\Omega} u_{t} \int_{\tau_{1}}^{\tau_{2}} \mid \mu_{1}(\varrho) z(x, 1, \varrho, t) d \varrho d x \\
= & \int_{\Omega} u_{t} f_{1}(u, v) d x .
\end{aligned}
$$


Similarly, multiplying equation $(2.8)_{2}$ by $v_{t}$ and integrating over $\Omega$, we obtain

$$
\begin{aligned}
& \frac{1}{2} \frac{d}{d t}\left[\left\|v_{t}\right\|^{2}+\mu\|\nabla v\|^{2}+(\lambda+\mu)\|\operatorname{div} v\|^{2}\right]+k_{1}\left\|v_{t}\right\|^{2} \\
& \quad+\int_{\Omega} v_{t} \int_{\tau_{1}}^{\tau_{2}} \mid \mu_{2}(\varrho) y(x, 1, \varrho, t) d \varrho d x \\
& =\int_{\Omega} v_{t} f_{2}(u, v) d x .
\end{aligned}
$$

Multiplying equation $(2.8)_{3}$ by $\left|\mu_{1}(\varrho)\right| z(x, \rho, \varrho, t)$ and integrating by parts over $\Omega \times(0,1) \times$ $\left(\tau_{1}, \tau_{2}\right)$, we obtain

$$
\begin{aligned}
& \int_{\Omega} \int_{0}^{1} \int_{\tau_{1}}^{\tau_{2}} \varrho\left|\mu_{1}(\varrho)\right| z(x, \rho, \varrho, t) z_{t}(x, \rho, \varrho, t) d \varrho d \rho d x \\
& \quad=-\int_{\Omega} \int_{0}^{1} \int_{\tau_{1}}^{\tau_{2}}\left|\mu_{1}(\varrho)\right| z(x, \rho, \varrho, t) z_{\rho}(x, \rho, \varrho, t) d \varrho d \rho d x
\end{aligned}
$$

therefore

$$
\begin{aligned}
\frac{d}{d t} & \frac{1}{2} \int_{\Omega} \int_{0}^{1} \int_{\tau_{1}}^{\tau_{2}} \varrho\left|\mu_{1}(\varrho)\right||z(x, \rho, \varrho, t)|^{2} d \varrho d \rho d x \\
& =-\frac{1}{2} \int_{\Omega} \int_{0}^{1} \int_{\tau_{1}}^{\tau_{2}}\left|\mu_{1}(\varrho)\right| \frac{d}{d \rho}|z(x, \rho, \varrho, t)|^{2} d \varrho d \rho d x \\
& =-\frac{1}{2} \int_{\Omega} \int_{\tau_{1}}^{\tau_{2}}\left|\mu_{1}(\varrho)\right||z(x, 1, \varrho, t)|^{2} d \varrho d x+\frac{1}{2} \int_{\Omega} \int_{\tau_{1}}^{\tau_{2}}\left|\mu_{1}(\varrho)\right|\left|u_{t}(x, t)\right|^{2} d \varrho d x
\end{aligned}
$$

Similarly, multiplying the fourth equation of $(2.8)$ by $\left|\mu_{2}(\varrho)\right| y(x, \rho, \varrho, t)$ and integrating over $\Omega \times(0,1) \times\left(\tau_{1}, \tau_{2}\right)$, we obtain

$$
\begin{aligned}
& \frac{d}{d t}\left(\frac{1}{2} \int_{\Omega} \int_{0}^{1} \int_{\tau_{1}}^{\tau_{2}} \varrho\left|\mu_{2}(\varrho)\right||y(x, \rho, \varrho, t)|^{2} d \varrho d \rho d x\right) \\
& \quad=-\frac{1}{2} \int_{\Omega} \int_{0}^{1} \int_{\tau_{1}}^{\tau_{2}}\left|\mu_{2}(\varrho)\right| \frac{d}{d \rho}|y(x, \rho, \varrho, t)|^{2} d \varrho d \rho d x \\
& \quad=-\frac{1}{2} \int_{\Omega} \int_{\tau_{1}}^{\tau_{2}}\left|\mu_{2}(\varrho)\right||y(x, 1, \varrho, t)|^{2} d \varrho d x+\frac{1}{2} \int_{\Omega} \int_{\tau_{1}}^{\tau_{2}}\left|\mu_{2}(\varrho)\right|\left|v_{t}(x, t)\right|^{2} d \varrho d x
\end{aligned}
$$

For the source term, we have

$$
\begin{aligned}
\int_{\Omega} & u_{t} f_{1}(u, v) d x+\int_{\Omega} v_{t} f_{2}(u, v) d x \\
= & \int_{\Omega} u_{t}\left(\alpha|u+v|^{p-1)}(u+v)+\beta|u|^{\frac{p-3}{2}} u|v|^{\frac{p+1}{2}}\right) \\
& +\int_{\Omega} v_{t}\left(\alpha|u+v|^{p-1}(u+v)+\beta|v|^{\frac{p-3}{2}} v|u|^{\frac{p+1}{2}}\right) \\
= & \int_{\Omega}\left(\alpha|u+v|^{p-1}(u+v)\left(u_{t}+v_{t}\right)+\beta\left(|u|^{\frac{p-3}{2}} u \cdot u_{t}\right)|v|^{\frac{p+1}{2}}\right. \\
& \left.+\beta\left(|v|^{\frac{p-3}{2}} v \cdot v_{t}\right)|u|^{\frac{p+1}{2}}\right) d x
\end{aligned}
$$




$$
=\frac{d}{d t} \int_{\Omega}\left(\frac{\alpha}{p+1}|u+v|^{p+1}+\frac{2 \beta}{p+1}|u v|^{\frac{p+1}{2}}\right) d x=\frac{d}{d t} \int_{\Omega} F(u, v) d x .
$$

By collecting the previous equations (2.13)-(2.17), we get

$$
\begin{aligned}
\frac{d}{d t} E(t)= & -k_{1}\left\|u_{t}\right\|^{2}-\int_{\Omega} u_{t} \int_{\tau_{1}}^{\tau_{2}} \mu_{1}(\varrho) z(x, 1, \varrho, t) d \varrho d x \\
& -k_{2}\left\|v_{t}\right\|^{2}-\int_{\Omega} v_{t} \int_{\tau_{1}}^{\tau_{2}} \mu_{2}(\varrho) y(x, 1, \varrho, t) d \varrho d x \\
& -\frac{1}{2} \int_{\Omega} \int_{\tau_{1}}^{\tau_{2}}\left|\mu_{1}(\varrho)\right||z(x, 1, \varrho, t)|^{2} d \varrho d x+\frac{1}{2}\left(\int_{\tau_{1}}^{\tau_{2}}\left|\mu_{1}(\varrho)\right| d \varrho\right)\left\|u_{t}\right\|^{2} \\
& -\frac{1}{2} \int_{\Omega} \int_{\tau_{1}}^{\tau_{2}}\left|\mu_{2}(\varrho)\right||y(x, 1, \varrho, t)|^{2} d \varrho d x+\frac{1}{2}\left(\int_{\tau_{1}}^{\tau_{2}}\left|\mu_{2}(\varrho)\right| d \varrho\right)\left\|v_{t}\right\|^{2} .
\end{aligned}
$$

Using Young's inequality, we obtain

$$
\begin{aligned}
& \int_{\Omega} u_{t} \int_{\tau_{1}}^{\tau_{2}} \mu_{1}(\varrho) z(x, 1, \varrho, t) d \varrho d x \\
& \quad \leq \frac{1}{2}\left(\int_{\tau_{1}}^{\tau_{2}}\left|\mu_{1}(\varrho)\right| d \varrho\right)\left\|u_{t}\right\|+\frac{1}{2} \int_{\Omega} \int_{\tau_{1}}^{\tau_{2}}\left|\mu_{1}(\varrho)\right||z(x, 1, \varrho, t)|^{2} d \varrho d x
\end{aligned}
$$

similarly

$$
\begin{aligned}
& \int_{\Omega} v_{t} \int_{\tau_{1}}^{\tau_{2}} \mu_{2}(\varrho) y(x, 1, \varrho, t) d \varrho d x \\
& \quad \leq \frac{1}{2}\left(\int_{\tau_{1}}^{\tau_{2}}\left|\mu_{2}(\varrho)\right| d \varrho\right)\left\|v_{t}\right\|+\frac{1}{2} \int_{\Omega} \int_{\tau_{1}}^{\tau_{2}}\left|\mu_{2}(\varrho)\right||y(x, 1, \varrho, t)|^{2} d \varrho d x
\end{aligned}
$$

This completes the proof.

\section{Global existence}

Theorem 3.1 (Global existence) Let $\left(u_{0}, v_{0}\right) \in\left(H^{2}(\Omega) \cap H_{0}^{1}(\Omega)\right)^{2},\left(u_{1}, v_{1}\right) \in\left(H_{0}^{1}(\Omega)\right)^{2}$ and $\left(f_{0}, g_{0}\right) \in\left(H^{1}\left(\Omega \times(0,1) \times\left(\tau_{1}, \tau_{2}\right)\right)\right)^{2}$ satisfying the compatibility condition

$$
\left(f_{0}(\cdot, 0), g_{0}(\cdot, 0)\right)=\left(u_{1}, v_{1}\right)
$$

Assume that (A1)-(A2) hold. Then, problem (2.8)-(2.9) admits a weak solution such that $u, v \in L^{\infty}\left(0, \infty ; H^{2}(\Omega) \cap H_{0}^{1}(\Omega)\right), u_{t}, v_{t} \in L^{\infty}\left(0, \infty ; H_{0}^{1}(\Omega)\right)$, and $u_{t t}, v_{t t} \in L^{2}\left(0, \infty, H_{0}^{1}(\Omega)\right)$.

Throughout this section we assume $\left(u_{0}, v_{0}\right) \in\left(H^{2}(\Omega) \cap H_{0}^{1}(\Omega)\right)^{2},\left(u_{1}, v_{1}\right) \in\left(H_{0}^{1}(\Omega)\right)^{2}$ and $f_{0}, g_{0} \in H^{2}\left(\Omega ; H^{1}(0,1)\right) \cap H_{0}^{1}\left(\Omega ; H^{1}(0,1)\right)$. We employ the Galerkin method to construct a global solution. Let $T>0$ be fixed and denote by $V_{k}$ the space generated by $\left\{w_{1}, w_{2}, \ldots, w_{k}\right\}$, where the set $\left\{w_{k}, k \in \mathbb{N}\right\}$ is a basis of $H^{2}(\Omega) \cap H_{0}^{1}(\Omega)$. Now, we define for $1 \leq j \leq k$ the sequence $\phi_{j}(x, \rho)$ as follows:

$$
\phi_{j}(x, 0)=w_{j}
$$


Then, we may extend $\phi_{j}(x, 0)$ by $\phi_{j}(x, \rho)$ over $L^{2}(\Omega \times[0,1])$ and denote by $Z_{k}$ the space generated by $\left\{\phi_{1}, \phi_{2}, \ldots, \phi_{k}\right\}$. We construct approximate solutions $\left(u^{k}, v^{k}, z^{k}, y^{k}\right) k=1,2, \ldots$. in the form

$$
\begin{aligned}
u^{k}(t) & =\sum_{j=1}^{k} g_{j k}(t) w_{j}(x), & z^{k}(t) & =\sum_{j=1}^{k} c_{j k}(t) \phi_{j}(x, \rho, \varrho) \\
v^{k}(t) & =\sum_{j=1}^{k} h_{j k}(t) w_{j}(x), & y^{k}(t) & =\sum_{j=1}^{k} d_{j k}(t) \phi_{j}(x, \rho, \varrho),
\end{aligned}
$$

where $g_{j k}, h_{j k}, c_{j k}$, and $d_{j k}, j=1,2, \ldots$, are determined by the following ordinary differential equations:

$$
\left\{\begin{array}{l}
\left\langle u_{t t}^{k}, w_{j}\right\rangle+\mu\left\langle\nabla u^{k}, \nabla w_{j}\right\rangle+(\lambda+\mu)\left\langle\operatorname{div} u^{k}, \operatorname{div} w_{j}\right\rangle+k_{1}\left\langle u_{t}^{k}, w_{j}\right\rangle \\
\quad+\left\langle\int_{\tau_{1}}^{\tau_{2}}\left|\mu_{1}(\varrho)\right| z^{k}(x, 1, \varrho, t) d \varrho, w_{j}\right\rangle=\left\langle f_{1}\left(u^{k}, v^{k}\right), w_{j}\right\rangle, \quad 1 \leq j \leq k, \\
\left\langle v_{t t}^{k}, w_{j}\right\rangle+\mu\left\langle\nabla v^{k}, \nabla w_{j}\right\rangle+(\lambda+\mu)\left\langle\operatorname{div} v^{k}, \operatorname{div} w_{j}\right\rangle+k_{2}\left\langle v_{t}^{k}, w_{j}\right\rangle \\
\quad+\left\langle\int_{\tau_{1}}^{\tau_{2}}\left|\mu_{2}(\varrho)\right| y^{k}(x, 1, \varrho, t) d \varrho, w_{j}\right\rangle=\left\langle f_{2}\left(u^{k}, v^{k}\right), w_{j}\right\rangle, \quad 1 \leq j \leq k, \\
\left\langle\varrho z_{t}^{k}+\frac{\partial}{\partial \rho} z^{k}, \phi_{j}\right\rangle=0, \quad 1 \leq j \leq k, \\
\left\langle\varrho y_{t}^{k}+\frac{\partial}{\partial \rho} y^{k}, \phi_{j}\right\rangle=0, \quad 1 \leq j \leq k,
\end{array}\right.
$$

and

$$
\left\{\begin{array}{l}
\left(u^{k}(0), u_{t, k}(0)\right)=\left(u_{0 k}, u_{1 k}\right), \\
\left(v^{k}(0), v_{t, k}(0)\right)=\left(u_{0 k}, u_{1 k}\right), \\
z^{k}(x, 0, \varrho, t)=u_{t}^{k}(x, t), \\
y^{k}(x, 0, \varrho, t)=v_{t}^{k}(x, t) .
\end{array}\right.
$$

Suppose that

$$
w_{j} \in H^{2}(\Omega) \text {. }
$$

We choose $u_{0}^{k}, v_{0}^{k}, u_{1}^{k}$ and $v_{1}^{k} \in\left[w_{1}, \ldots, w_{k}\right]$, where

$$
\begin{aligned}
& u^{k}(0)=u_{0}^{k}=\sum_{j=1}^{k}\left(u_{0}, w_{j}\right) w_{j} \rightarrow u_{0} \quad \text { in } H^{2}(\Omega) \cap H_{0}^{1}(\Omega) \text { as } k \rightarrow+\infty, \\
& u_{t}^{k}(0)=u_{1}^{k}=\sum_{j=1}^{k}\left(u_{1}, w_{j}\right) w_{j} \rightarrow u_{1} \quad \text { in } H_{0}^{1}(\Omega) \text { as } k \rightarrow+\infty, \\
& v^{k}(0)=v_{0}^{k}=\sum_{j=1}^{k}\left(v_{0}, w_{j}\right) w_{j} \rightarrow v_{0} \quad \text { in } H^{2}(\Omega) \cap H_{0}^{1}(\Omega) \text { as } k \rightarrow+\infty, \\
& v_{t}^{k}(0)=v_{1}^{k}=\sum_{j=1}^{k}\left(v_{1}, w_{j}\right) w_{j} \rightarrow v_{1} \quad \text { in } H_{0}^{1}(\Omega) \text { as } k \rightarrow+\infty, \\
& z^{k}(\rho, 0)=z_{0}^{k}=\sum_{j=1}^{k}\left(f_{0}, \phi_{j}\right) \phi_{j} \rightarrow f_{0} \quad \text { in } L^{2}\left(\Omega \times(0,1) \times\left(\tau_{1}, \tau_{2}\right)\right) \text { as } k \rightarrow+\infty,
\end{aligned}
$$




$$
y^{k}(\rho, 0)=y_{0}^{k}=\sum_{j=1}^{k}\left(g_{0}, \phi_{j}\right) \phi_{j} \rightarrow g_{0} \quad \text { in } L^{2}\left(\Omega \times(0,1) \times\left(\tau_{1}, \tau_{2}\right)\right) \text { as } k \rightarrow+\infty \text {. }
$$

By virtue of the theory of ordinary differential equations, system (3.4)-(3.12) has a unique local solution which is extended to a maximal interval $\left[0, T_{k}\left[\right.\right.$ (with $0<T_{k} \leq+\infty$ ). We can utilize a standard compactness argument for the limiting procedure.

The first estimate.

Lemma 3.2 There exists a constant $T>0$ such that the approximate solution satisfies, for all $k \geq 1$ :

$$
\begin{aligned}
& u^{k}, v^{k} \quad \text { are bounded in } L^{\infty}\left(0, T ; H_{0}^{1}(\Omega)\right), \\
& u_{t}^{k}, v_{t}^{k} \quad \text { are bounded in } L^{\infty}\left(0, T ; L^{2}(\Omega)\right), \\
& z^{k}(x, \rho, \varrho, t), y^{k}(x, \rho, \varrho, t) \quad \text { are bounded in } L^{\infty}\left(0, T ; L^{2}\left(\Omega \times(0,1) \times\left(\tau_{1}, \tau_{2}\right)\right)\right) .
\end{aligned}
$$

Proof Multiplying the first and second equations of (3.4) by $\left(g_{j k}^{\prime}\right)$ and $\left(h_{j k}^{\prime}\right)$ respectively and summing with respect to $j$, we obtain

$$
\begin{aligned}
& \frac{1}{2} \frac{d}{d t}\left[\left\|u_{t}^{k}\right\|^{2}+\mu\left\|\nabla u^{k}\right\|^{2}+(\lambda+\mu)\left\|\operatorname{div} u^{k}\right\|^{2}+\left\|v_{t}^{k}\right\|^{2}\right. \\
& \left.\quad+\mu\left\|\nabla v^{k}\right\|^{2}+(\lambda+\mu)\left\|\operatorname{div} v^{k}\right\|^{2}\right] \\
& \quad+\int_{\Omega} u_{t}^{k} \int_{\tau_{1}}^{\tau_{2}} \mu_{1}(\varrho) z^{k}(x, 1, \varrho, t) d \varrho d x+\int_{\Omega} v_{t}^{k} \int_{\tau_{1}}^{\tau_{2}} \mu_{2}(\varrho) y^{k}(x, 1, \varrho, t) d \varrho d x \\
& \quad+k_{1}\left\|u_{t}^{k}\right\|^{2}+k_{2}\left\|v_{t}^{k}\right\|^{2}=\left\langle f_{1}\left(u^{k}, v^{k}\right), u_{t}^{k}\right\rangle+\left\langle f_{2}\left(u^{k}, v^{k}\right), v_{t}^{k}\right\rangle, \quad 1 \leq j \leq k .
\end{aligned}
$$

Multiplying (3.4) by $\left|\mu_{1}(\varrho)\right|\left(c_{j k}\right)$ and $\left|\mu_{2}(\varrho)\right|\left(d_{j k}\right)$ respectively, iterating over $\Omega \times(0,1) \times$ $\left(\tau_{1}, \tau_{2}\right)$, and summing with respect to $j$, we obtain

$$
\begin{aligned}
\frac{1}{2} \frac{d}{d t} \int_{\Omega} \int_{0}^{1} \int_{\tau_{1}}^{\tau_{2}} \varrho\left|\mu_{1}(\varrho)\right|\left|z^{k}\right|^{2} d \varrho d \rho d x \\
\quad+\frac{1}{2} \frac{d}{d t} \int_{\Omega} \int_{0}^{1} \int_{\tau_{1}}^{\tau_{2}} \varrho\left|\mu_{2}(\varrho)\right|\left|y^{k}\right|^{2} d \varrho d \rho d x \\
=-\frac{1}{2} \int_{\Omega} \int_{\tau_{1}}^{\tau_{2}} \varrho\left|\mu_{1}(\varrho)\right|\left|z^{k}(x, 1, \varrho, t)\right|^{2} d \varrho d x+\frac{1}{2}\left(\int_{\tau_{1}}^{\tau_{2}}\left|\mu_{1}(\varrho)\right| d \varrho\right)\left\|u_{t}^{k}\right\|^{2} \\
\quad-\frac{1}{2} \int_{\Omega} \int_{0}^{1} \int_{\tau_{1}}^{\tau_{2}} \varrho\left|\mu_{2}(\varrho)\right|\left|y^{k}(x, 1, \varrho, t)\right|^{2} d \varrho d x+\frac{1}{2}\left(\int_{\tau_{1}}^{\tau_{2}}\left|\mu_{2}(\varrho)\right| d \varrho\right)\left\|v_{t}^{k}\right\|^{2} .
\end{aligned}
$$

By summing (3.16)-(3.17), we have

$$
\begin{aligned}
\frac{1}{2} \frac{d}{d t}[ & \left.\left\|u_{t}^{k}\right\|^{2}+\mu\left\|\nabla u^{k}\right\|^{2}+(\lambda+\mu)\left\|\operatorname{div} u_{k}\right\|^{2}+\left\|v_{t}^{k}\right\|^{2}+\mu\left\|\nabla v^{k}\right\|^{2}(\lambda+\mu)\left\|\operatorname{div} v^{k}\right\|^{2}\right] \\
& +\frac{1}{2} \frac{d}{d t}\left[\int_{\Omega} \int_{0}^{1} \int_{\tau_{1}}^{\tau_{2}} \varrho\left|\mu_{1}(\varrho) \| z^{k}(x, \rho, \varrho, t)\right|^{2} d \varrho d \rho d x\right. \\
& \left.+\int_{\Omega} \int_{0}^{1} \int_{\tau_{1}}^{\tau_{2}} \varrho\left|\mu_{2}(\varrho) \| y^{k}(x, \rho, \varrho, t)\right|^{2} d \varrho d \rho d x\right]
\end{aligned}
$$




$$
\begin{aligned}
& +k_{1}\left\|u_{t}^{k}\right\|^{2}+k_{2}\left\|v_{t}^{k}\right\|^{2} \\
& +\frac{1}{2} \int_{\Omega} \int_{\tau_{1}}^{\tau_{2}}\left|\mu_{1}(\varrho)\right|\left|z^{k}(x, 1, \varrho, t)\right|^{2} d \varrho d x-\frac{1}{2}\left(\int_{\tau_{1}}^{\tau_{2}}\left|\mu_{1}(\varrho)\right| d \varrho\right)\left\|u_{t}^{k}\right\|^{2} \\
& +\frac{1}{2} \int_{\Omega} \int_{\tau_{1}}^{\tau_{2}}\left|\mu_{2}(\varrho)\right|\left|y^{k}(x, 1, \varrho, t)\right|^{2} d \varrho d x-\frac{1}{2}\left(\int_{\tau_{1}}^{\tau_{2}}\left|\mu_{2}(\varrho)\right| d \varrho\right)\left\|v_{t}^{k}\right\|^{2} \\
& =\int_{\Omega}\left(u_{t}^{k} f_{1}\left(u^{k}, v^{k}\right)+v_{t}^{k} f_{2}\left(u^{k}, v^{k}\right)\right) d x \\
& -\int_{\Omega} u_{t}^{k} \int_{\tau_{1}}^{\tau_{2}} \mu_{1}(\varrho) z_{k}(x, 1, \varrho, t) d \varrho d x-\int_{\Omega} v_{t}^{k} \int_{\tau_{1}}^{\tau_{2}} \mu_{2}(\varrho) y_{k}(x, 1, \varrho, t) d \varrho d x .
\end{aligned}
$$

By using Holder and Young's inequalities, we have

$$
\begin{aligned}
& \frac{1}{2} \frac{d}{d t}\left[\left\|u_{t}^{k}\right\|^{2}+\mu\left\|\nabla u^{k}\right\|^{2}+(\lambda+\mu)\left\|\operatorname{div} u^{k}\right\|^{2}\right. \\
& \left.\quad+\left\|v_{t}^{k}\right\|^{2}+\mu\left\|\nabla v^{k}\right\|^{2}+(\lambda+\mu)\left\|\operatorname{div} v^{k}\right\|^{2}\right] \\
& \quad+\frac{1}{2} \frac{d}{d t} \int_{0}^{1} \int_{\tau_{1}}^{\tau_{2}} \varrho\left[\left|\mu_{1}(\varrho)\right|\left\|z^{k}(x, \rho, \varrho, t)\right\|^{2}\right. \\
& \left.\quad+\varrho\left|\mu_{2}(\varrho)\right|\left\|y^{k}(x, \rho, \varrho, t)\right\|^{2}\right] d \varrho d \rho \\
& \quad+\left[k_{1}-\left(\int_{\tau_{1}}^{\tau_{2}}\left|\mu_{1}(\varrho)\right| d \varrho\right)\right]\left\|u_{t}^{k}\right\|^{2}+\left[k_{2}-\left(\int_{\tau_{1}}^{\tau_{2}}\left|\mu_{2}(\varrho)\right| d \varrho\right)\right]\left\|v_{t}^{k}\right\|^{2} \\
& \leq \int_{\Omega}\left(u_{t}^{k} f_{1}\left(u^{k}, v^{k}\right)+v_{t}^{k} f_{2}\left(u^{k}, v^{k}\right)\right) d x .
\end{aligned}
$$

Integrating over $(0, t), 0<t<T_{k}$, we obtain

$$
\begin{aligned}
& \left\|u_{t}^{k}(t)\right\|^{2}+\mu\left\|\nabla u^{k}(t)\right\|^{2}+(\lambda+\mu)\left\|\operatorname{div} u^{k}(t)\right\|^{2}+\left\|v_{t}^{k}(t)\right\|^{2} \\
& +\mu\left\|\nabla v^{k}(t)\right\|^{2}+(\lambda+\mu)\left\|\operatorname{div} v^{k}(t)\right\|^{2} \\
& +\int_{0}^{1} \int_{\tau_{1}}^{\tau_{2}} \varrho\left[\left|\mu_{1}(\varrho)\right|\left\|z^{k}(x, \rho, \varrho, t)\right\|^{2}+\varrho\left|\mu_{2}(\varrho)\right|\left\|y^{k}(x, \rho, \varrho, t)\right\|^{2}\right] d \varrho d \rho \\
& +2\left[k_{1}-\left(\int_{\tau_{1}}^{\tau_{2}}\left|\mu_{1}(\varrho)\right| d \varrho\right)\right] \int_{0}^{t}\left\|u_{t}^{k}(s)\right\|^{2} d s \\
& +2\left[k_{2}-\left(\int_{\tau_{1}}^{\tau_{2}}\left|\mu_{2}(\varrho)\right| d \varrho\right)\right] \int_{0}^{t}\left\|v_{t}^{k}(s)\right\|^{2} d s \\
& \leq C_{0}+c \int_{0}^{t} \int_{\Omega}\left[u_{t}^{k} f_{1}\left(u^{k}, v^{k}\right)+v_{t}^{k} f_{2}\left(u^{k}, v^{k}\right)\right] d x d s,
\end{aligned}
$$

where

$$
\begin{aligned}
C_{0}= & C\left(\left\|u_{0}^{k}\right\|_{H^{1}(\Omega)},\left\|v_{0}^{k}\right\|_{H^{1}(\Omega)},\left\|u_{1}^{k}\right\|_{L^{2}(\Omega)},\left\|v_{1}^{k}\right\|_{L^{2}(\Omega)},\right. \\
& \left.\left\|f_{0}\right\|_{L^{2}\left(\Omega \times(0,1) \times\left(\tau_{1}, \tau_{2}\right)\right)},\left\|g_{0}\right\|_{L^{2}\left(\Omega \times(0,1) \times\left(\tau_{1}, \tau_{2}\right)\right)}\right)
\end{aligned}
$$


is a positive constant. We just need to estimate the right-hand terms of (3.20). Applying Hölder's inequality, Sobolev's embedding theorem, and Young's inequality, we obtain

$$
\begin{aligned}
\left|\int_{0}^{t} \int_{\Omega} u_{t}^{k}(s) f_{1}\left(u^{k}(s), v^{k}(s)\right) d s\right| \\
\leq C \int_{0}^{t} \int_{\Omega}\left(\left|u^{k}(s)\right|^{p}+\left|v^{k}(s)\right|^{p}+\left|u^{k}(s)\right|^{\frac{p-1}{2}}\left|v^{k}(s)\right|^{\frac{p+1}{2}}\right)\left|u_{t}^{k}(s)\right| d s \\
\leq C \int_{0}^{t} \int_{\Omega}\left(\left\|u^{k}(s)\right\|_{2 p}^{p}+\left\|v^{k}(s)\right\|_{2 p}^{p}\right. \\
\left.\quad+\left\|u^{k}(s)\right\|_{3(p-1)}^{\frac{p-1}{2}}\left\|v^{k}(s)\right\|_{\frac{3(p+1)}{2}}^{\frac{p+1}{2}}\right)\left\|u_{t}^{k}(s)(s)\right\|_{2} d s \\
\leq C \int_{0}^{t} \int_{\Omega}\left(\left\|\nabla u^{k}(s)\right\|_{2}^{p}+\left\|\nabla v^{k}(s)\right\|_{2}^{p}\right. \\
\left.\quad+\left\|\nabla u^{k}(s)\right\|_{2}^{\frac{p-1}{2}}\left\|\nabla v^{k}(s)\right\|_{2}^{\frac{p+1}{2}}\right)\left\|u_{t}^{k}(s)(s)\right\|_{2} d s \\
\leq C \int_{0}^{t} \int_{\Omega}\left(\left\|u_{t}^{k}(s)\right\|_{2}^{2}+\left\|\nabla u^{k}(s)\right\|_{2}^{2 p}+\left\|\nabla v^{k}(s)\right\|_{2}^{2 p}\right. \\
\left.\quad+\left\|\nabla u^{k}(s)\right\|_{2}^{(p-1)}\left\|\nabla v^{k}(s)\right\|_{2}^{(p+1)}\right) d s,
\end{aligned}
$$

when we have used in (3.21) the Sobolev imbedding in (2.5) and the fact when $n=3$ then $2 p=3(p-1)=\frac{3(p+1)}{2}=6$.

Likewise, we obtain

$$
\begin{aligned}
& \left|\int_{0}^{t} \int_{\Omega} v_{t}^{k}(s) f_{2}\left(u^{k}(s), v^{k}(s)\right) d s\right| \\
& \quad \leq C \int_{0}^{t} \int_{\Omega}\left(\left\|v_{t}^{k}(s)\right\|_{2}^{2}+\left\|\nabla u^{k}(s)\right\|_{2}^{2 p}+\left\|\nabla v^{k}(s)\right\|_{2}^{2 p}\right. \\
& \left.\quad+\left\|\nabla u^{k}(s)\right\|_{2}^{p+1}\left\|\nabla v^{k}(s)\right\|_{2}^{p-1}\right) d s .
\end{aligned}
$$

Let

$$
X_{k}(t)=\left\|u_{t}^{k}(t)\right\|_{2}^{2}+\left\|v_{t}^{k}(t)\right\|_{2}^{2}+\left\|\nabla u^{k}(t)\right\|_{2}^{2}+\left\|\nabla v^{k}(t)\right\|_{2}^{2} .
$$

From assumptions of Lemma 2.1, we can find positive constants such that

$$
\begin{aligned}
X_{k}(t) & +c_{1}\left\|\operatorname{div} u^{k}(t)\right\|^{2}+c_{1}\left\|\operatorname{div} v^{k}(t)\right\|^{2} \\
& +c_{2} \int_{0}^{1} \int_{\tau_{1}}^{\tau_{2}} \varrho\left[\left|\mu_{1}(\varrho)\right|\left\|z^{k}(x, \rho, \varrho, t)\right\|^{2}+\varrho\left|\mu_{2}(\varrho)\right|\left\|y^{k}(x, \rho, \varrho, t)\right\|^{2}\right] d \varrho d \rho \\
& +c_{3} \int_{0}^{t}\left\|u_{t}^{k}(s)\right\|^{2} d s+c_{3} \int_{0}^{t}\left\|v_{t}^{k}(s)\right\|^{2} d s \\
\leq & C_{0}+c \int_{0}^{t} \int_{\Omega}\left(X_{k}(t)\right)^{p} d x d s .
\end{aligned}
$$

Particulary, we have

$$
X_{k}(t) \leq C_{0}+c \int_{0}^{t} \int_{\Omega}\left(X_{k}(t)\right)^{p} d x d s
$$


Using Gronwall-type inequality, we can get

$$
X_{k}(t) \leq\left[C_{0}-(p-1) C t\right]^{-1 /(p-1)} .
$$

Thus, we deduce from (3.26) that there exists a time $T>0$ such that

$$
X_{k}(t) \leq C_{1}, \quad \forall t \in[0, T],
$$

where $C_{1}$ is a positive constant independent of $k$. Then inequality (3.27) established the first two parts of lemma. The last part of lemma immediately follows from (3.24).

The second estimate: First, we are going to estimate $u_{t t}^{k}(0)$ and $v_{t t}^{k}(0)$. Testing the first and second equations in (3.4) by $g_{j, k}^{\prime \prime}(t)$ and $h_{j, k}^{\prime \prime}(t)$ respectively and taking $t=0$, we obtain

$$
\begin{aligned}
& \left\|u_{t t}^{k}(0)\right\|_{2}^{2}+\left\|v_{t t}^{k}(0)\right\|_{2}^{2} \\
& \leq\left\|\Delta u_{0}^{k}\right\|_{2}^{2}+\left\|\Delta v_{0}^{k}\right\|_{2}^{2}+c\left\|\operatorname{div} u_{0}^{k}\right\|_{2}+c\left\|\operatorname{div} v_{0}^{k}\right\|_{2}^{2}+\left\|\Delta u_{t}^{k}(0)\right\|_{2}^{2} \\
& \quad+\left\|\Delta v_{t}^{k}(0)\right\|_{2}^{2}+c \int_{\Omega} \int_{\tau_{1}}^{\tau_{2}}\left|\mu_{1}(\varrho) \| \Delta z^{k}(x, 1, \varrho, 0)\right|^{2} d \varrho d x \\
& \quad+c \int_{\Omega} \int_{\tau_{1}}^{\tau_{2}}\left|\mu_{2}(\varrho) \| \Delta y^{k}(x, 1, \varrho, 0)\right|^{2} d \varrho d x .
\end{aligned}
$$

From (3.7)-(3.12), we have

$$
\left\|u_{t t}^{k}(0)\right\|_{2}^{2}+\left\|v_{t t}^{k}(0)\right\|_{2}^{2} \leq C
$$

In order to calculate the second estimate, we take the derivatives of the first and second equations of system (3.4) with respect to $t$, we get

$$
\begin{aligned}
& \left\langle u_{t t t}^{k}, w_{j}\right\rangle+\mu\left\langle\nabla u_{t}^{k}, \nabla w_{j}\right\rangle+(\lambda+\mu)\left\langle\operatorname{div} u_{t}^{k}, \operatorname{div} w_{j}\right\rangle \\
& \quad+k_{1}\left\langle u_{t t}^{k}, w_{j}\right\rangle+\left\langle\int_{\tau_{1}}^{\tau_{2}}\left|\mu_{1}(\varrho)\right| z_{t}^{k}(x, 1, \varrho, t) d \varrho, w_{j}\right\rangle=\left\langle D f_{1}\left(u_{k}, v_{k}\right), w_{j}\right\rangle, \quad 1 \leq j \leq k
\end{aligned}
$$

and

$$
\begin{aligned}
& \left\langle v_{t t t}^{k}, w_{j}\right\rangle+\mu\left\langle\nabla v_{t}^{k}, \nabla w_{j}\right\rangle+(\lambda+\mu)\left\langle\operatorname{div} v_{t}^{k}, \operatorname{div} w_{j}\right\rangle \\
& \quad+k_{2}\left\langle v_{t t}^{k}, w_{j}\right\rangle+\left\langle\int_{\tau_{1}}^{\tau_{2}}\left|\mu_{2}(\varrho)\right| y_{t}^{k}(x, 1, \varrho, t) d \varrho, w_{j}\right\rangle=\left\langle D f_{2}\left(u_{k}, v_{k}\right), w_{j}\right\rangle, \quad 1 \leq j \leq k .
\end{aligned}
$$

Multiplying by $\left(g_{j, k}^{\prime \prime}(t)\right)$ and $\left(h_{j, k}^{\prime \prime}(t)\right)$ respectively and summing with respect to $j$ from 1 to $k$, we obtain

$$
\begin{aligned}
& \frac{1}{2} \frac{d}{d t}\left[\left\|u_{t t}^{k}(t)\right\|^{2}+\mu\left\|\nabla u_{t}^{k}(t)\right\|^{2}+(\lambda+\mu)\left\|\operatorname{div} u_{t}^{k}(t)\right\|^{2}\right]+k_{1}\left\|u_{t t}^{k}(t)\right\|^{2} \\
& \quad+\int_{\Omega} u_{t t}^{k}(t) \int_{\tau_{1}}^{\tau_{2}}\left|\mu_{1}(\varrho)\right| z_{t}^{k}(x, 1, \varrho, t) d \varrho d x \\
& =\int_{\Omega} D f_{1}\left(u_{k}, v_{k}\right) u_{t t}^{k}(t) d x, \quad 1 \leq j \leq k
\end{aligned}
$$


and

$$
\begin{gathered}
\frac{1}{2} \frac{d}{d t}\left[\left\|v_{t t}^{k}\right\|^{2}+\mu\left\|\nabla v_{t}^{k}\right\|^{2}+(\lambda+\mu)\left\|\operatorname{div} v_{t}^{k}\right\|^{2}\right]+k_{2}\left\|v_{t t}^{k}\right\|^{2} \\
\quad+\int_{\Omega} v_{t t}^{k}(t) \int_{\tau_{1}}^{\tau_{2}}\left|\mu_{2}(\varrho)\right| y_{t}^{k}(x, 1, \varrho, t) d \varrho d x \\
=\int_{\Omega} D f_{2}\left(u_{k}, v_{k}\right) v_{t t}^{k}(t) d x, \quad 1 \leq j \leq k
\end{gathered}
$$

Differentiating the third and fourth equations in (3.4) with respect to $t$, we get

$$
\begin{aligned}
& \left\langle\varrho z_{t t}^{k}+\frac{\partial}{\partial \rho} z_{t}^{k}, \phi_{j}\right\rangle=0, \quad 1 \leq j \leq k \\
& \left\langle\varrho y_{t t}^{k}+\frac{\partial}{\partial \rho} y_{t}^{k}, \phi_{j}\right\rangle=0, \quad 1 \leq j \leq k .
\end{aligned}
$$

Multiplying by $\left|\mu_{1}(\varrho)\right| c_{j k}^{\prime}$ and $\left|\mu_{2}(\varrho)\right| d_{j k}^{\prime}$ respectively, integrating over $(0,1) \times\left(\tau_{1}, \tau_{2}\right)$, and summing over $j$ from 1 to $k$, it follows that

$$
\begin{aligned}
& \frac{1}{2} \frac{d}{d t} \int_{0}^{1} \int_{\tau_{1}}^{\tau_{2}} \int_{\Omega} \varrho\left|\mu_{1}(\varrho)\right|\left|z_{t}^{k}(x, \rho, \varrho, t)\right|^{2} d \varrho d \rho d x \\
& \quad+\frac{1}{2} \int_{0}^{1} \int_{\Omega}\left|\mu_{1}(\varrho)\right| \frac{d}{d \rho}\left|z_{t}^{k}(x, \rho, \varrho, t)\right|^{2} d \varrho d \rho d x=0, \\
& \frac{1}{2} \frac{d}{d t} \int_{0}^{1} \int_{\tau_{1}}^{\tau_{2}} \int_{\Omega} \varrho\left|\mu_{1}(\varrho)\right|\left|y_{t}^{k}(x, \rho, \varrho, t)\right|^{2} d \varrho d \rho d x \\
& \quad+\frac{1}{2} \int_{0}^{1} \int_{\Omega}\left|\mu_{2}(\varrho)\right| \frac{d}{d \rho}\left|y_{t}^{k}(x, \rho, \varrho, t)\right|^{2} d \varrho d \rho d x=0,
\end{aligned}
$$

then we obtain

$$
\begin{aligned}
\frac{1}{2} & \frac{d}{d t} \int_{0}^{1} \int_{\Omega} \int_{\tau_{1}}^{\tau_{2}} \varrho\left|\mu_{1}(\varrho)\right|\left|z_{t}^{k}(x, \rho, \varrho, t)\right|^{2} d \varrho d \rho d x \\
& +\frac{1}{2} \int_{\Omega} \int_{\tau_{1}}^{\tau_{2}}\left|\mu_{1}(\varrho)\right|\left|z_{t}^{k}(x, 1, \varrho, t)\right|^{2} d \varrho d x \\
& -\frac{1}{2}\left(\int_{\tau_{1}}^{\tau_{2}}\left|\mu_{1}(\varrho)\right| d \varrho\right)\left\|u_{t t}^{k}(t)\right\|^{2} d x=0
\end{aligned}
$$

and

$$
\begin{aligned}
\frac{1}{2} & \frac{d}{d t} \int_{0}^{1} \int_{\tau_{1}}^{\tau_{2}} \int_{\Omega} \varrho\left|\mu_{2}(\varrho)\right|\left|y_{t}^{k}(x, \rho, \varrho, t)\right|^{2} d \varrho d \rho d x \\
& +\frac{1}{2} \int_{\Omega} \int_{\tau_{1}}^{\tau_{2}}\left|\mu_{2}(\varrho)\right|\left|y_{t}^{k}(x, 1, \varrho, t)\right|^{2} d \varrho d x \\
& -\frac{1}{2}\left(\int_{\tau_{1}}^{\tau_{2}}\left|\mu_{2}(\varrho)\right| d \varrho\right)\left\|v_{t t}^{k}(t)\right\|^{2} d x=0 .
\end{aligned}
$$


Taking the sum of (3.30), (3.31),(3.32), and (3.33), we get

$$
\begin{aligned}
\frac{1}{2} \frac{d}{d t}[ & \left.\left\|u_{t t}^{k}\right\|^{2}+\mu\left\|\nabla u_{t}^{k}\right\|^{2}+(\lambda+\mu)\left\|\operatorname{div} u_{t}^{k}\right\|^{2}+\left\|v_{t t}^{k}\right\|^{2}+\mu\left\|\nabla v_{t}^{k}\right\|^{2}+(\lambda+\mu)\left\|\operatorname{div} v_{t}^{k}\right\|^{2}\right] \\
& +\frac{1}{2} \frac{d}{d t}\left[\int_{0}^{1} \int_{\Omega} \int_{\tau_{1}}^{\tau_{2}} \varrho\left|\mu_{1}(\varrho)\right|\left|z_{t}^{k}(x, \rho, \varrho, t)\right|^{2} d \varrho d \rho d x\right. \\
& \left.+\int_{0}^{1} \int_{\tau_{1}}^{\tau_{2}} \int_{\Omega} \varrho\left|\mu_{2}(\varrho)\right|\left|y_{t}^{k}(x, \rho, \varrho, t)\right|^{2} d \varrho d \rho d x\right] \\
& +k_{1}\left\|u_{t t}^{k}\right\|^{2}+k_{2}\left\|v_{t t}^{k}\right\|^{2}+\frac{1}{2} \int_{\Omega} \int_{\tau_{1}}^{\tau_{2}}\left|\mu_{1}(\varrho)\right|\left|z_{t}^{k}(x, 1, \varrho, t)\right|^{2} d \varrho d x \\
& +\frac{1}{2} \int_{\Omega}^{\tau_{\tau_{1}}}\left|\mu_{2}(\varrho)\right|\left|y_{t}^{k}(x, 1, \varrho, t)\right|^{2} d \varrho d x \\
= & \frac{1}{2}\left(\int_{\tau_{1}}^{\tau_{2}}\left|\mu_{1}(\varrho)\right| d \varrho\right)\left\|u_{t t}^{k}(t)\right\|^{2}+\frac{1}{2}\left(\int_{\tau_{1}}^{\tau_{2}}\left|\mu_{2}(\varrho)\right| d \varrho\right)\left\|v_{t t}^{k}(t)\right\|^{2} \\
& -\int_{\Omega} u_{t t}^{k}(t) \int_{\tau_{1}}^{\tau_{2}}\left|\mu_{1}(\varrho)\right| z_{t}^{k}(x, 1, \varrho, t) d \varrho d x \\
& -\int_{\Omega} v_{t t}^{k}(t) \int_{\tau_{1}}^{\tau_{2}}\left|\mu_{2}(\varrho)\right| y_{t}^{k}(x, 1, \varrho, t) d \varrho d x \\
& +\int_{\Omega} D f_{1}\left(u_{k}, v_{k}\right) u_{t t}^{k}(t) d x+\int_{\Omega} D f_{2}\left(u_{k}, v_{k}\right) v_{t t}^{k}(t) d x .
\end{aligned}
$$

Using Cauchy-Schwarz and Young's inequalities, we conclude

$$
\begin{aligned}
\left|\int_{\Omega} u_{t t}^{k}(t) \int_{\tau_{1}}^{\tau_{2}}\right| \mu_{1}(\varrho)\left|z_{t}^{k}(x, 1, \varrho, t) d \varrho d x\right| \\
\leq \frac{1}{2}\left(\int_{\tau_{1}}^{\tau_{2}}\left|\mu_{1}(\varrho)\right| d \varrho\right)\left\|u_{t t}^{k}(t)\right\|^{2} \\
\quad+\frac{1}{2} \int_{\tau_{1}}^{\tau_{2}}\left|\mu_{1}(\varrho)\right|\left\|z_{t}^{k}(x, 1, \varrho, t)\right\|^{2} d \varrho .
\end{aligned}
$$

Similarly,

$$
\begin{aligned}
\left|\int_{\Omega} v_{t t}^{k}(t) \int_{\tau_{1}}^{\tau_{2}}\right| \mu_{2}(\varrho)\left|y_{t}^{k}(x, 1, \varrho, t) d \varrho d x\right| \\
\leq \frac{1}{2}\left(\int_{\tau_{1}}^{\tau_{2}}\left|\mu_{2}(\varrho)\right| d \varrho\right)\left\|v_{t t}^{k}(t)\right\|^{2} \\
\quad+\frac{1}{2} \int_{\tau_{1}}^{\tau_{2}}\left|\mu_{2}(\varrho)\right|\left\|y_{t}^{k}(x, 1, \varrho, t)\right\|^{2} d \varrho .
\end{aligned}
$$

For the source term

$$
\begin{aligned}
& \int_{\Omega} D f_{1}\left(u_{k}, v_{k}\right) u_{t t}^{k}(t) d x \\
& \quad \leq C\left[\left(\left\|u^{k}\right\|^{p-1}+\left\|v^{k}\right\|^{p-1}\right)\left\|u_{t}^{k}\right\|+\left(\left\|u^{k}\right\|^{p-1}+\left\|v^{k}\right\|^{p-1}\right)\left\|v_{t}^{k}\right\|\right]\left\|u_{t t}^{k}\right\| \\
& \quad \leq C\left[\left\|u^{k}\right\|_{2}^{2(p-1)}+\left\|v^{k}\right\|_{2}^{2(p-1)}+\left\|u_{t}^{k}\right\|_{2}^{2}+\left\|v_{t}^{k}\right\|_{2}^{2}\right]\left\|u_{t t}^{k}\right\|_{2}
\end{aligned}
$$




$$
\leq\left\|u_{t t}^{k}(t)\right\|_{2}^{2}+c
$$

and

$$
\begin{aligned}
& \int_{\Omega} D f_{2}\left(u_{k}, v_{k}\right) v_{t t}^{k}(t) d x \\
& \quad \leq C\left[\left(\left\|u^{k}\right\|^{p-1}+\left\|v^{k}\right\|^{p-1}\right)\left\|u_{t}^{k}\right\|+\left(\left\|u^{k}\right\|^{p-1}+\left\|v^{k}\right\|^{p-1}\right)\left\|v_{t}^{k}\right\|\right]\left\|v_{t t}^{k}\right\| \\
& \quad \leq C\left[\left\|u^{k}\right\|_{2}^{2(p-1)}+\left\|v^{k}\right\|_{2}^{2(p-1)}+\left\|u_{t}^{k}\right\|_{2}^{2}+\left\|v_{t}^{k}\right\|_{2}^{2}\right]\left\|v_{t t}^{k}\right\|_{2} \\
& \quad \leq\left\|v_{t t}^{k}(t)\right\|_{2}^{2}+c .
\end{aligned}
$$

Taking into account (3.35)-(3.38) into (3.34), we get

$$
\begin{aligned}
\frac{1}{2} \frac{d}{d t}[ & \left.\left\|u_{t t}^{k}\right\|^{2}+\mu\left\|\nabla u_{t}^{k}\right\|^{2}+(\lambda+\mu)\left\|\operatorname{div} u_{t}^{k}\right\|^{2}+\left\|v_{t t}^{k}\right\|^{2}+\mu\left\|\nabla v_{t}^{k}\right\|^{2}+(\lambda+\mu)\left\|\operatorname{div} v_{t}^{k}\right\|^{2}\right] \\
& +\frac{1}{2} \frac{d}{d t}\left[\int_{0}^{1} \int_{\Omega} \int_{\tau_{1}}^{\tau_{2}} \varrho\left|\mu_{1}(\varrho)\right|\left|z_{t}^{k}(x, \varrho, \rho, t)\right|^{2} d \varrho d \rho d x\right. \\
& \left.+\frac{1}{2} \int_{0}^{1} \int_{\tau_{1}}^{\tau_{2}} \int_{\Omega} \varrho\left|\mu_{1}(\varrho) \| y_{t}^{k}(x, \varrho, \rho, t)\right|^{2} d \varrho d \rho d x\right] \\
& +k_{1}\left\|u_{t t}^{k}\right\|^{2}+k_{2}\left\|v_{t t}^{k}\right\|^{2}+\frac{1}{2} \int_{\tau_{1}}^{\tau_{2}}\left|\mu_{1}(\varrho)\right|\left\|z_{t}^{k}(x, 1, \varrho, t)\right\|^{2} d \varrho \\
& +\frac{1}{2} \int_{\tau_{1}}^{\tau_{2}}\left|\mu_{2}(\varrho)\right|\left\|y_{t}^{k}(x, 1, \varrho, t)\right\|^{2} d \varrho \\
\leq & \frac{1}{2}\left(\int_{\tau_{1}}^{\tau_{2}}\left|\mu_{1}(\varrho)\right| d \varrho\right)\left\|u_{t t}^{k}(t)\right\|^{2}+\frac{1}{2}\left(\int_{\tau_{1}}^{\tau_{2}}\left|\mu_{2}(\varrho)\right| d \varrho\right)\left\|v_{t t}^{k}(t)\right\|^{2} \\
& +\left(\frac{\int_{\tau_{1}}^{\tau_{2}}\left|\mu_{1}(\varrho)\right| d \varrho}{2}\right)\left\|u_{t t}^{k}\right\|^{2}+\left(\frac{\int_{\tau_{1}}^{\tau_{2}}\left|\mu_{2}(\varrho)\right| d \varrho}{2}\right)\left\|v_{t t}^{k}\right\|^{2} \\
& +\frac{1}{2} \int_{\tau_{1}}^{\tau_{2}}\left|\mu_{1}(\varrho)\right|\left\|z_{t}^{k}(x, 1, \varrho, t)\right\|^{2} d \varrho+\frac{1}{2} \int_{\tau_{1}}^{\tau_{2}}\left|\mu_{2}(\varrho)\right|\left\|y_{t}^{k}(x, 1, \varrho, t)\right\|^{2} d \varrho \\
& +\left\|u_{t t}^{k}(t)\right\|^{2}+\left\|v_{t t}^{k}(t)\right\|^{2}+c .
\end{aligned}
$$

After simplification, we obtain

$$
\begin{aligned}
& \frac{1}{2} \frac{d}{d t}\left[\left\|u_{t t}^{k}\right\|^{2}+\mu\left\|\nabla u_{t}^{k}\right\|^{2}+(\lambda+\mu)\left\|\operatorname{div} u_{t}^{k}\right\|^{2}+\left\|v_{t t}^{k}\right\|^{2}+\mu\left\|\nabla v_{t}^{k}\right\|^{2}+(\lambda+\mu)\left\|\operatorname{div} v_{t}^{k}\right\|^{2}\right] \\
& \quad+\frac{1}{2} \frac{d}{d t}\left[\int_{0}^{1} \int_{\Omega} \int_{\tau_{1}}^{\tau_{2}} \varrho\left|\mu_{1}(\varrho) \| z_{t}^{k}(x, \varrho, \rho, t)\right|^{2} d \varrho d \rho d x\right. \\
& \left.\quad+\frac{1}{2} \int_{0}^{1} \int_{\tau_{1}}^{\tau_{2}} \int_{\Omega} \varrho\left|\mu_{1}(\varrho) \| y_{t}^{k}(x, \varrho, \rho, t)\right|^{2} d \varrho d \rho d x\right] \\
& \quad+k_{1}\left\|u_{t t}^{k}\right\|^{2}+k_{2}\left\|v_{t t}^{k}\right\|^{2} \\
& \leq\left(\int_{\tau_{1}}^{\tau_{2}}\left|\mu_{1}(\varrho)\right| d \varrho\right)\left\|u_{t t}^{k}(t)\right\|^{2}+\left(\int_{\tau_{1}}^{\tau_{2}}\left|\mu_{2}(\varrho)\right| d \varrho\right)\left\|v_{t t}^{k}(t)\right\|^{2} \\
& \quad+\left\|u_{t t}^{k}(t)\right\|^{2}+\left\|v_{t t}^{k}(t)\right\|^{2}+c .
\end{aligned}
$$


Integrating (3.39) over $(0, t)$, we get

$$
\begin{aligned}
& {\left[\left\|u_{t t}^{k}(t)\right\|^{2}+\mu\left\|\nabla u_{t}^{k}(t)\right\|^{2}+(\lambda+\mu)\left\|\operatorname{div} u_{t}^{k}(t)\right\|^{2}+\left\|v_{t t}^{k}(t)\right\|^{2}\right.} \\
& \left.+\mu\left\|\nabla v_{t}^{k}(t)\right\|^{2}+(\lambda+\mu)\left\|\operatorname{div} v_{t}^{k}(t)\right\|^{2}\right] \\
& \times\left[\int_{0}^{1} \int_{\Omega} \int_{\tau_{1}}^{\tau_{2}} \varrho\left|\mu_{1}(\varrho)\right|\left|z_{t}^{k}(x, \varrho, \rho, t)\right|^{2} d \varrho d \rho d x\right. \\
& \left.+\frac{1}{2} \int_{0}^{1} \int_{\tau_{1}}^{\tau_{2}} \int_{\Omega} \varrho\left|\mu_{1}(\varrho)\right|\left|y_{t}^{k}(x, \varrho, \rho, t)\right|^{2} d \varrho d \rho d x\right] \\
& +\left(k_{1}-\left(\int_{\tau_{1}}^{\tau_{2}}\left|\mu_{1}(\varrho)\right| d \varrho\right)\right) \int_{0}^{t}\left\|u_{t t}^{k}(s)\right\|^{2} d s \\
& +\left(k_{2}-\left(\int_{\tau_{1}}^{\tau_{2}}\left|\mu_{2}(\varrho)\right| d \varrho\right)\right) \int_{0}^{t}\left\|v_{t t}^{k}(s)\right\|^{2} d s \\
& \leq\left[\left\|u_{t t}^{k}(0)\right\|^{2}+\mu\left\|\nabla u_{t}^{k}(0)\right\|^{2}+(\lambda+\mu)\left\|\operatorname{div} u_{t}^{k}(0)\right\|^{2}+\left\|v_{t t}^{k}(0)\right\|^{2}\right. \\
& \left.+\mu\left\|\nabla v_{t}^{k}(0)\right\|^{2}+(\lambda+\mu)\left\|\operatorname{div} v_{t}^{k}(0)\right\|^{2}\right] \\
& \times\left[\int_{0}^{1} \int_{\Omega} \int_{\tau_{1}}^{\tau_{2}} \varrho\left|\mu_{1}(\varrho)\right|\left|z_{t}^{k}(x, \varrho, \rho, 0)\right|^{2} d \varrho d \rho d x\right. \\
& \left.+\frac{1}{2} \int_{0}^{1} \int_{\tau_{1}}^{\tau_{2}} \int_{\Omega} \varrho\left|\mu_{1}(\varrho)\right|\left|y_{t}^{k}(x, \varrho, \rho, 0)\right|^{2} d \varrho d \rho d x\right] \\
& +\int_{0}^{t}\left\|u_{t t}^{k}(s)\right\|^{2} d s+\int_{0}^{t}\left\|v_{t t}^{k}(s)\right\|^{2} d s+c
\end{aligned}
$$

Taking

$$
y^{k}(t)=\left\|u_{t t}^{k}(t)\right\|^{2}+\left\|v_{t t}^{k}(t)\right\|^{2}
$$

and by using Gronwall's inequality, we conclude that

$$
\begin{aligned}
& u_{t}^{k} \text { and } v_{t}^{k} \text { are bounded in } L^{\infty}\left(0, T ; H_{0}^{1}(\Omega)\right), \\
& u_{t t}^{k} \text { and } v_{t t}^{k} \text { are bounded in } L^{\infty}\left(0, T ; L^{2}(\Omega)\right), \\
& z_{t}^{k} \text { and } y_{t}^{k} \text { are bounded in } L^{\infty}\left(0, T ; L^{2}\left(\Omega \times(0,1) \times\left(\tau_{1}, \tau_{2}\right)\right)\right) .
\end{aligned}
$$

Applying Dunford-Pettis' theorem, we deduce from (3.13), (3.14), (3.15), (3.40), (3.41), and (3.42), replacing the sequence $u_{k}$ with a subsequence if necessary, that

$$
\begin{aligned}
& u^{k} \rightarrow u, v^{k} \rightarrow v \text { weak-star in } L^{\infty}\left(0, T ; H_{0}^{1}(\Omega)\right), \\
& z^{k} \rightarrow z, y^{k} \rightarrow y \text { weak-star in } L^{\infty}\left(0, T ; L^{2}\left(\Omega \times(0,1) \times\left(\tau_{1}, \tau_{2}\right)\right)\right), \\
& u_{t}^{k} \rightarrow u_{t}, v_{t}^{k} \rightarrow v_{t} \text { weak-star in } L^{\infty}\left(0, T ; H_{0}^{1}(\Omega)\right), \\
& u_{t t}^{k} \rightarrow u_{t t}, v_{t t}^{k} \rightarrow v_{t t} \text { weak-star in } L^{\infty}\left(0, T ; L^{2}(\Omega)\right), \\
& z_{t}^{k} \rightarrow z_{t}, y_{t}^{k} \rightarrow y_{t} \text { weak-star in } L^{\infty}\left(0, T ; L^{2}\left(\Omega \times(0,1) \times\left(\tau_{1}, \tau_{2}\right)\right)\right) .
\end{aligned}
$$


Corollary 3.3 The sequences of approximate solutions $\left\{u_{k}, u_{k}\right\}$ satisfy, as $k \rightarrow \infty$,

$$
\left\{\begin{array}{l}
f_{1}\left(u_{k}, v_{k}\right) \rightarrow f_{1}(u, v) \text { strongly in } L^{\infty}\left(0, T ; L^{2}(\Omega)\right), \\
f_{2}\left(u_{k}, v_{k}\right) \rightarrow f_{2}(u, v) \text { strongly in } L^{\infty}\left(0, T ; L^{2}(\Omega)\right) .
\end{array}\right.
$$

Proof The proof is similar to that of [11].

We can complete the proof of theorem as in [2].

\section{General decay}

In this section we prove that the solution of problem (2.8)-(2.9) decays generally to a trivial solution using the energy method and a suitable Lyapunov functional.

In the following, we present our main stability result.

Theorem 4.1 The solution of (2.8) satisfies, for two positive constants $\alpha, \beta$, the estimate

$$
E(t) \leq \beta e^{-\alpha t}, \quad \forall t \geq 0
$$

To prove the desired result, we create a Lyapunov functional equivalent to $E$. For this, we define some functions that allow us to construct this Lyapunov function.

Lemma 4.2 Let $(u, v, z, y)$ be a solution of problem (2.8). Then the functional

$$
\varphi(t)=\int_{\Omega} u(t) u_{t}(t) d x+\int_{\Omega} v(t) v_{t}(t) d x
$$

satisfies the estimate

$$
\begin{aligned}
\varphi^{\prime}(t) \leq & \left(1+\frac{C k_{1}^{2}}{\mu}\right)\left\|u_{t}(t)\right\|^{2}+\left(1+\frac{C k_{2}}{\mu}\right)\left\|v_{t}(t)\right\|^{2}-\frac{\mu}{2}\|\nabla u(t)\|^{2}-\frac{\mu}{2}\|\nabla v(t)\|^{2} \\
& -(\lambda+\mu)\|\operatorname{div} u(t)\|^{2}-(\lambda+\mu)\|\operatorname{div} v(t)\|^{2} \\
& +\frac{C k_{1}}{\mu} \int_{\Omega} \int_{\tau_{1}}^{\tau_{2}}\left|\mu_{1}(\varrho) \| \nabla z(x, 1, \varrho, t)\right|^{2} d \varrho d x \\
& +\frac{C k_{2}}{\mu} \int_{\Omega} \int_{\tau_{1}}^{\tau_{2}}\left|\mu_{2}(\varrho) \| \nabla y(x, 1, \varrho, t)\right|^{2} d \varrho d x \\
& +(p+1) \int_{\Omega} F(u(t), v(t)) d x .
\end{aligned}
$$

Proof Taking the derivative of (4.2), we obtain

$$
\varphi^{\prime}(t)=\int_{\Omega}\left|u_{t}(t)\right|^{2} d x+\int_{\Omega} u(t) u_{t t}(t) d x+\int_{\Omega}\left|v_{t}(t)\right|^{2} d x+\int_{\Omega} v(t) v_{t t}(t) d x .
$$


From problem (2.8) and using integration by parts, we get

$$
\begin{aligned}
\varphi^{\prime}(t)= & \left\|u_{t}(t)\right\|^{2}+\left\|v_{t}(t)\right\|^{2} \\
& +\int_{\Omega} u(t)\left(\Delta_{e} u-k_{1} u_{t}-\int_{\tau_{1}}^{\tau_{2}} \mu_{1}(\varrho) z(x, 1, \varrho, t) d \varrho+f_{1}(u, v)\right) d x \\
& +\int_{\Omega} v(t)\left(\Delta_{e} v-k_{2} \Delta v_{t}-\int_{\tau_{1}}^{\tau_{2}} \mu_{2}(\varrho) \Delta y(x, 1, \varrho, t) d \varrho+f_{2}(u, v)\right) d x \\
= & \left\|u_{t}(t)\right\|^{2}+\left\|v_{t}(t)\right\|^{2}-k_{1} \int_{\Omega} u u_{t} d x-k_{2} \int_{\Omega} v v_{t} d x \\
& -\mu\|\nabla u(t)\|^{2}-(\lambda+\mu)\|\operatorname{div} u(t)\|^{2}-\mu\|\nabla v(t)\|^{2}-(\lambda+\mu)\|\operatorname{div} v(t)\|^{2} \\
& -\int_{\Omega} u(t) \int_{\tau_{1}}^{\tau_{2}} \mu_{1}(\varrho) z(x, 1, \varrho, t) d \varrho-\int_{\Omega} v(t) \int_{\tau_{1}}^{\tau_{2}} \mu_{2}(\varrho) y(x, 1, \varrho, t) d \varrho \\
& +\int_{\Omega} v(t) f_{2}(u, v) d x+\int_{\Omega} u(t) f_{1}(u, v) d x .
\end{aligned}
$$

By using Hölder's and Young's inequalities, we have

$$
\begin{aligned}
& \int_{\Omega} u(t) \int_{\tau_{1}}^{\tau_{2}} \mu_{1}(\varrho) z(x, 1, \varrho, t) d \varrho d x \\
& \leq \frac{\varepsilon}{2}\|u(t)\|^{2}+\frac{1}{2 \varepsilon} \int_{\Omega}\left(\int_{\tau_{1}}^{\tau_{2}}\left|\mu_{1}(\varrho)\right| z(x, 1, \varrho, t) d \varrho\right)^{2} d x \\
& \leq \frac{C \varepsilon}{2}\|\nabla u(t)\|^{2}+\frac{1}{2 \varepsilon}\left(\int_{\tau_{1}}^{\tau_{2}}\left|\mu_{1}(\varrho)\right| d \varrho\right) \int_{\Omega} \int_{\tau_{1}}^{\tau_{2}}\left|\mu_{1}(\varrho)\right||\nabla z(x, 1, \varrho, t)|^{2} d \varrho \\
& \leq \frac{C \varepsilon}{2}\|\nabla u(t)\|^{2}+\frac{k_{1}}{2 \varepsilon} \int_{\Omega} \int_{\tau_{1}}^{\tau_{2}}\left|\mu_{1}(\varrho)\right||z(x, 1, \varrho, t)|^{2} d \varrho .
\end{aligned}
$$

Similarly,

$$
\begin{aligned}
& \int_{\Omega} v(t) \int_{\tau_{1}}^{\tau_{2}} \mu_{1}(\varrho) y(x, 1, \varrho, t) d \varrho d x \\
& \quad \leq \frac{C \varepsilon}{2}\|\nabla v(t)\|^{2}+\frac{k_{2}}{2 \varepsilon} \int_{\Omega} \int_{\tau_{1}}^{\tau_{2}}\left|\mu_{1}(\varrho)\right||y(x, 1, \varrho, t)|^{2} d \varrho .
\end{aligned}
$$

Young's and Poincaré's inequalities give

$$
k_{1} \int_{\Omega} u(t) u_{t}(t) d x \leq \frac{C \varepsilon}{2}\|\nabla u(t)\|^{2}+\frac{k_{1}^{2}}{2 \varepsilon}\left\|u_{t}(t)\right\|^{2}
$$

and

$$
k_{2} \int_{\Omega} v(t) \nabla v_{t}(t) d x \leq \frac{C \varepsilon}{2}\|\nabla v(t)\|^{2}+\frac{k_{2}^{2}}{2 \varepsilon}\left\|v_{t}(t)\right\|^{2} .
$$

For the source term, we have

$$
\int_{\Omega} u(t) f_{1}(u, v) d x+\int_{\Omega} v(t) f_{2}(u, v) d x=(p+1) \int_{\Omega} F(u, v) d x
$$

Combining equations (4.4)-(4.9) and taking $\varepsilon=\frac{\mu}{2 C}$, thus, our proof is completed. 
Lemma 4.3 Let $(u, v, z, y)$ be a solution of problem (2.8). Then the functional

$$
I(t)=\int_{\Omega} \int_{0}^{1} \int_{\tau_{1}}^{\tau_{2}} \varrho e^{-\varrho \rho}\left[\left|\mu_{1}(\varrho)\right||z(x, \rho, \varrho, t)|^{2}+\left|\mu_{2}(\varrho)\right||y(x, \rho, \varrho, t)|^{2}\right] d x d \varrho d \rho
$$

satisfies the estimate

$$
\begin{aligned}
I^{\prime}(t) \leq & -e^{-\tau_{2}} \int_{\Omega} \int_{\tau_{1}}^{\tau_{2}}\left|\mu_{1}(\varrho)\right||z(x, 1, \varrho, t)|^{2} d x d \varrho \\
& -e^{-\tau_{2}} \int_{\Omega} \int_{\tau_{1}}^{\tau_{2}}\left|\mu_{2}(\varrho)\right||y(x, 1, \varrho, t)|^{2} d x d \varrho \\
& +k_{1}\left\|u_{t}(t)\right\|^{2}+k_{2}\left\|v_{t}(t)\right\|^{2}-I(t) .
\end{aligned}
$$

Proof Differentiating (4.10) with respect to $t$, we get

$$
\begin{aligned}
\frac{d}{d t} I(t)= & 2 \int_{\Omega} \int_{0}^{1} \int_{\tau_{1}}^{\tau_{2}} \varrho e^{-\varrho \rho}\left|\mu_{1}(\varrho)\right| z(x, \rho, \varrho, t) z_{t}(x, \rho, \varrho, t) d x d \varrho d \rho \\
& +2 \int_{\Omega} \int_{0}^{1} \int_{\tau_{1}}^{\tau_{2}} \varrho e^{-\varrho \rho}\left|\mu_{2}(\varrho)\right| y(x, \rho, \varrho, t) y_{t}(x, \rho, \varrho, t) d x d \varrho d \rho .
\end{aligned}
$$

By using (2.6)-(2.7), we have

$$
\begin{aligned}
\frac{d}{d t} I(t)= & -\int_{\Omega} \int_{0}^{1} \int_{\tau_{1}}^{\tau_{2}} e^{-\varrho \rho}\left[\left|\mu_{1}(\varrho)\right| \frac{\partial}{\partial \rho}|z(x, \rho, \varrho, t)|^{2}\right. \\
& \left.+\left|\mu_{2}(\varrho)\right| \frac{\partial}{\partial \rho}|y(x, \rho, \varrho, t)|^{2}\right] d x d \varrho d \rho \\
= & -\int_{\Omega} \int_{0}^{1} \int_{\tau_{1}}^{\tau_{2}}\left[\left|\mu_{1}(\varrho)\right| \frac{\partial}{\partial \rho}\left(e^{-\varrho \rho}|z(x, \rho, \varrho, t)|^{2}\right)\right. \\
& \left.+\left|\mu_{2}(\varrho)\right| \frac{\partial}{\partial \rho}\left(e^{-\varrho \rho}|y(x, \rho, \varrho, t)|^{2}\right)\right] d x d \varrho d \rho \\
& -\int_{\Omega} \int_{0}^{1} \int_{\tau_{1}}^{\tau_{2}} \varrho e^{-\varrho \rho}\left[\left|\mu_{1}(\varrho)\right||z(x, \rho, \varrho, t)|^{2}+\left|\mu_{2}(\varrho)\right||y(x, \rho, \varrho, t)|^{2}\right] d x d \varrho d \rho .
\end{aligned}
$$

Thus,

$$
\begin{aligned}
\frac{d}{d t} I(t)= & -\int_{\Omega} \int_{\tau_{1}}^{\tau_{2}} e^{-\varrho}\left|\mu_{1}(\varrho)\right||z(x, 1, \varrho, t)|^{2} d x d \varrho+\left(\int_{\tau_{1}}^{\tau_{2}}\left|\mu_{1}(\varrho)\right| d \varrho\right)\left\|u_{t}(x, \varrho, t)\right\|^{2} \\
& -\int_{\Omega} \int_{\tau_{1}}^{\tau_{2}} e^{-\varrho}\left|\mu_{2}(\varrho)\right||y(x, 1, \varrho, t)|^{2} d x d \varrho+\left(\int_{\tau_{1}}^{\tau_{2}}\left|\mu_{2}(\varrho)\right| d \varrho\right)\left\|v_{t}(x, \varrho, t)\right\|^{2} \\
& -\int_{\Omega} \int_{0}^{1} \int_{\tau_{1}}^{\tau_{2}} \varrho e^{-\varrho \rho}\left[\left|\mu_{1}(\varrho)\right||z(x, \rho, \varrho, t)|^{2}+\left|\mu_{2}(\varrho)\right||y(x, \rho, \varrho, t)|^{2}\right] d x d \varrho d \rho .
\end{aligned}
$$

Since $e^{-\varrho}$ is a deceasing function over $\left(\tau_{1}, \tau_{2}\right)$, the desired estimate (4.11) follows immediately from (2.11).

Proof of Theorem 4.1 Now, we define the following functional:

$$
\mathcal{F}(t)=N E(t)+\varphi(t)+N_{2} I(t)
$$


where $N$ and $N_{2}$ are positive constants. It is easy to prove $\mathcal{F}(t)$ and $E(t)$ are equivalent, namely there exist two positive constants $\kappa_{1}$ and $\kappa_{2}$ such that

$$
\kappa_{1} E(t) \leq \mathcal{F}(t) \leq \kappa_{2} E(t) .
$$

From Lemmas 4.2 and 4.3, we have, for any $t \geq 0$,

$$
\begin{aligned}
\mathcal{F}^{\prime}(t) \leq & -N\left[k_{1}-\left(\int_{\tau_{1}}^{\tau_{2}}\left|\mu_{1}(\varrho)\right| d \varrho\right)\right]\left\|u_{t}\right\|^{2}-N\left[k_{2}-\left(\int_{\tau_{1}}^{\tau_{2}}\left|\mu_{2}(\varrho)\right| d \varrho\right)\right]\left\|v_{t}\right\|^{2} \\
& +\left(1+\frac{C k_{1}^{2}}{\mu}\right)\left\|u_{t}(t)\right\|^{2}+\left(1+\frac{C k_{2}}{\mu}\right)\left\|v_{t}(t)\right\|^{2}-\frac{\mu}{2}\|\nabla u(t)\|^{2}-\frac{\mu}{2}\|\nabla v(t)\|^{2} \\
& -(\lambda+\mu)\|\operatorname{div} u(t)\|^{2}-(\lambda+\mu)\|\operatorname{div} v(t)\|^{2} \\
& +\frac{C k_{1}}{\mu} \int_{\Omega} \int_{\tau_{1}}^{\tau_{2}}\left|\mu_{1}(\varrho) \| \nabla z(x, 1, \varrho, t)\right|^{2} d \varrho d x \\
& +\frac{C k_{2}}{\mu} \int_{\Omega} \int_{\tau_{1}}^{\tau_{2}}\left|\mu_{2}(\varrho) \| \nabla y(x, 1, \varrho, t)\right|^{2} d \varrho d x \\
& +(p+1) \int_{\Omega} F(u(t), v(t)) d x \\
& -N_{2} e^{-\tau_{2}} \int_{\Omega} \int_{\tau_{1}}^{\tau_{2}}\left|\mu_{1}(\varrho) \| z(x, 1, \varrho, t)\right|^{2} d x d \varrho \\
& -N_{2} e^{-\tau_{2}} \int_{\Omega} \int_{\tau_{1}}^{\tau_{2}}\left|\mu_{2}(\varrho) \| y(x, 1, \varrho, t)\right|^{2} d x d \varrho \\
& -N_{2} \int_{\Omega} \int_{0}^{1} \int_{\tau_{1}}^{\tau_{2}} \varrho e^{-\varrho \rho}\left[\left|\mu_{1}(\varrho) \| z(x, \rho, \varrho, t)\right|^{2}\right. \\
& \left.+\left|\mu_{2}(\varrho) \| y(x, \rho, \varrho, t)\right|^{2}\right] d x d \varrho d \rho \\
& +N_{2} k_{1}\left\|u_{t}(t)\right\|^{2}+N_{2} k_{2}\left\|v_{t}(t)\right\|^{2} .
\end{aligned}
$$

Thus

$$
\begin{aligned}
\mathcal{F}^{\prime}(t) \leq & -\left(N \sigma_{1}-\left(1+\frac{C k_{1}^{2}}{\mu}\right)-N_{2} k_{1}\right)\left\|u_{t}\right\|^{2}-\left(N \sigma_{2}-\left(1+\frac{C k_{2}^{2}}{\mu}\right)-N_{2} k_{2}\right)\left\|v_{t}\right\|^{2} \\
& -\frac{\mu}{2}\|\nabla u(t)\|^{2}-\frac{\mu}{2}\|\nabla v(t)\|^{2}-(\lambda+\mu)\|\operatorname{div} u(t)\|^{2}-(\lambda+\mu)\|\operatorname{div} v(t)\|^{2} \\
& -\left(N_{2} e^{-\tau_{2}}-\frac{C k_{1}}{\mu}\right) \int_{\Omega} \int_{\tau_{1}}^{\tau_{2}}\left|\mu_{1}(\varrho)\right||z(x, 1, \varrho, t)|^{2} d \varrho d x \\
& -\left(N_{2} e^{-\tau_{2}}-\frac{C k_{2}}{\mu}\right) \int_{\Omega} \int_{\tau_{1}}^{\tau_{2}}\left|\mu_{2}(\varrho)\right||y(x, 1, \varrho, t)|^{2} d \varrho d x \\
& +(p+1) \int_{\Omega} F(u(t), v(t)) d x \\
& -N_{2} \int_{\Omega} \int_{0}^{1} \int_{\tau_{1}}^{\tau_{2}} \varrho e^{-\varrho \rho}\left[\left|\mu_{1}(\varrho) \| z(x, \rho, \varrho, t)\right|^{2}\right. \\
& \left.+\left|\mu_{2}(\varrho) \| y(x, \rho, \varrho, t)\right|^{2}\right] d x d \varrho d \rho,
\end{aligned}
$$


where

$$
\sigma_{1}=\left[k_{1}-\left(\int_{\tau_{1}}^{\tau_{2}}\left|\mu_{1}(\varrho)\right| d \varrho\right)\right], \quad \sigma_{2}=\left[k_{2}-\left(\int_{\tau_{1}}^{\tau_{2}}\left|\mu_{2}(\varrho)\right| d \varrho\right)\right] .
$$

First, we take $N_{2}$ large such that

$$
N_{2} e^{-\tau_{2}}-\frac{C k_{1}}{\mu}>0 \text { and } N_{2} e^{-\tau_{2}}-\frac{C k_{2}}{\mu}>0 .
$$

We choose $N>0$ large enough so that

$$
N \sigma_{1}-\left(1+\frac{C k_{1}^{2}}{\mu}\right)-N_{2} k_{1}>0
$$

and

$$
N \sigma_{2}-\left(1+\frac{C k_{2}^{2}}{\mu}\right)-N_{2} k_{2}>0
$$

and $\mathcal{F} \sim E$. Thus we arrive at

$$
\mathcal{F}^{\prime}(t) \leq-\alpha \mathcal{F}(t)
$$

which yields, by integration,

$$
\mathcal{F}(t) \leq \mathcal{F}(0) e^{-\alpha t}, \quad \forall t \geq 0
$$

The use of $\mathcal{F} \sim E$ gives

$$
E(t) \leq \beta e^{-\alpha t}, \quad \forall t \geq 0 .
$$

\section{Acknowledgements}

The first author would like to thank all the professors of the mathematics department at the University of Annaba in Algeria, especially his Professors/Scientists Pr. Mohamed Haiour, Pr. Ahmed-Salah Chibi, and Pr. Azzedine Benchettah for the important content of masters and PhD courses in pure and applied mathematics which he received during his studies. Moreover, he thanks them for the additional help they provided to him during office hours in their office about the few concepts/difficulties he had encountered, and he appreciates their talent and dedication for their postgraduate students currently and previously. In addition, the idea and research project in this paper was presented by the first author (Pr. Salah Boulaaras) and was carried out by both authors. The authors are grateful to the anonymous referees for the careful reading and their important observations/suggestions for the sake of improving this paper.

\section{Funding}

Not applicable.

Availability of data and materials

Not applicable.

Ethics approval and consent to participate

Not applicable.

\section{Competing interests}

The authors declare that there is no conflict of interests regarding the publication of this manuscript. The authors declare that they have no competing interests.

\section{Consent for publication}

Not applicable. 
Authors' contributions

The authors contributed equally in this article. They have all read and approved the final manuscript.

\section{Author details}

'Department of Mathematics, College of Sciences and Arts, ArRas, Qassim University, Buraidah, Kingdom of Saudi Arabia. ${ }^{2}$ Laboratory of Fundamental and Applied Mathematics of Oran (LMFAO), University of Oran 1, Ahmed Benbella, Oran, Algeria. ${ }^{3}$ Laboratory of Operator Theory and PDEs: Foundations and Applications, Department of Mathematics, Faculty of Exact Sciences, University of El Oued, Box. 789, El Oued, 39000, Algeria.

\section{Publisher's Note}

Springer Nature remains neutral with regard to jurisdictional claims in published maps and institutional affiliations.

Received: 3 October 2020 Accepted: 20 November 2020 Published online: 30 November 2020

\section{References}

1. Mezouar, N., Boulaaras, S.: Global existence and exponential decay of solutions for generalized coupled non-degenerate Kirchhoff system with a time varying delay term. Bound. Value Probl. 2020, 90 (2020). https://doi.org/10.1186/s13661-020-01390-9

2. Boulaaras, S., Guefaifia, R., Mezouar, N., Alghamdi, A.M.: Global existence and decay for a system of two singular nonlinear viscoelastic equations with general source and localized frictional damping terms. J. Funct. Spaces 2020 Article ID 5085101 (2020). https://doi.org/10.1155/2020/5085101

3. Ouchenane, D., Boulaaras, S., Alharbi, A., Cherif, B.: Blow up of coupled nonlinear Klein-Gordon system with distributed delay, strong damping, and source terms. J. Funct. Spaces 2020, Article ID 5297063 (2020). https://doi.org/10.1155/2020/5297063

4. Doudi, N., Boulaaras, S.: Global existence combined with general decay of solutions for coupled Kirchhoff system with a distributed delay term. Rev. R. Acad. Cienc. Exactas Fís. Nat., Ser. A Mat. 114, 204 (2020). https://doi.org/10.1007/s13398-020-00938-9

5. Gala, S., Ragusa, M.A.: Logarithmically improved regularity criterion for the Boussinesq equations in Besov spaces with negative indices. Appl. Anal. 95(6), 1271-1279 (2016)

6. Gala, S., Liu, Q., Ragusa, M.A.: A new regularity criterion for the nematic liquid crystal flows. Appl. Anal. 91(9), $1741-1747$ (2012)

7. Guliyev, V.S., Guliyev, R.V., Omarova, M.N., Ragusa, M.A.: Schrödinger type operators on local generalized Morrey spaces related to certain nonnegative potentials. Discrete Contin. Dyn. Syst., Ser. B 25(2), 671-690 (2020)

8. Lui, G.: Well-posedness and exponential decay of solutions for a transmission problem with distributed delay. Electron. J. Differ. Equ. 2017(174), 1 (2017)

9. Wu, S.-T.: On decay and blow up of solutions for a system of nonlinear wave equations. J. Math. Anal. Appl. 394 360-377 (2012)

10. Mezouar, N., Boulaaras, S.: Global existence and decay of solutions of a singular nonlocal viscoelastic system with damping terms. Topol. Methods Nonlinear Anal. 56(1), 283-312 (2020). https://doi.org/10.12775/TMNA.2020.014

11. Xiaosen, H., Mingxin, W.: Global existence and blow-up of solutions for a system of nonlinear viscoelastic wave equations with damping and source. Nonlinear Anal. 71, 5427-5450 (2009)

12. Bchatnia, A., Daoulatli, M.: Behavior of the energy for Lamé systems in bounded domains with nonlinear damping and external force. Electron. J. Differ. Equ. 2013(1), 1 (2013)

13. Beniani, A., Taouaf, N., Benaissa, A.: Well-posedness and exponential stability for coupled Lamé system with viscoelastic term and strong damping. Comput. Math. Appl. 75(12), 4397-4404 (2018)

14. Boulaaras, S., Ouchenane, D.: General decay for a coupled Lamé system of nonlinear viscoelastic equations. Math. Methods Appl. Sci. 43(4), 1717-1735 (2020)

15. Boulaaras, S.: A well-posedness and exponential decay of solutions for a coupled Lamé system with viscoelastic term and logarithmic source terms. Appl. Anal. (2019). https://doi.org/10.1080/00036811.2019.1648793

16. Bchatnia, A., Guesmia, A.: Well-posedness and asymptotic stability for the Lamé system with infinite memories in a bounded domain. Math. Control Relat. Fields 4(4), 451-463 (2014)

17. Taouaf, N., Amroun, N., Benaissa, A., Beniani, A.: Well-posedness and asymptotic stability for the Lamé system with internal distributed delay. Math. Morav. 22(1), 31-41 (2018)

18. Feng, B., Hajjej, Z., Balegh, M.: Existence and general decay rate estimates of a coupled Lamé system only with viscoelastic dampings. Math. Methods Appl. Sci. 1(18) (2020, in press). https://doi.org/10.1002/mma.6586 